\title{
Neural Network Classification Method for Aircraft in ISAR Images
}

\author{
Krasimir Slavyanov \\ Department Computer Systems and \\ Technologies \\ "Vasil Levski" National Military Uni- \\ versity \\ Shumen, Bulgaria \\ k.o.slavyanov@gmail.com
}

\begin{abstract}
This article offers a neural network method for automatic classification of Inverse Synthetic Aperture Radar objects represented in images with high level of postreceive optimization. A full explanation of the procedures of two-layer neural network architecture creating and training is described. The classification in the recognition stage is proposed, based on several main classes or sets of flying objects. The classification sets are designed according to distinctive specifications in the structural models of the aircrafts. The neural network is experimentally simulated in MATLAB environment. Numerical results of the experiments carried, prove the correct classification of the objects in ISAR optimized images.
\end{abstract}

Keywords- artificial neural network, engine position, reference model.

\section{INTRODUCTION}

For the Inverse Synthetic Aperture Radar Systems (ISAR) the main purpose of the observed object's image is to be further classified and fully recognized as a type, model, specifications and owner even if the friend-foe system onboard or radio communications at all are not operational or used properly. In order to provide easy access to the ISAR principles of work a special dedicated airplane flight and ISAR simulation systems can be used to provide the environment for the ISAR system improvements ([1], [2], [3], [4], [5]).

Of particular importance for the proper recognition of objects in ISAR images is the classification of objects in the images according to particular details ([6], [7], [8], [9]). Several approaches can be used to analyse different data obtained for flying objects. The more successful classifications of the object, so they can be successfully merged with fuzzy logic tools to enable full recognition of objects according to specific rules [10].

\section{MATERIALS AND METHODS}

Preconditions and design.

For the purposes of the study, an ISAR simulation approach has been used ([1], [2], [6], [11]) in order to generate simulated ISAR images with additive Gaussian noise with constant zero mean and variance 0.01 and "salt and pepper" noise with density 0.015 (figure 1).

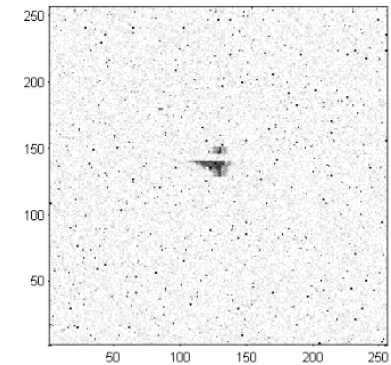

(a)

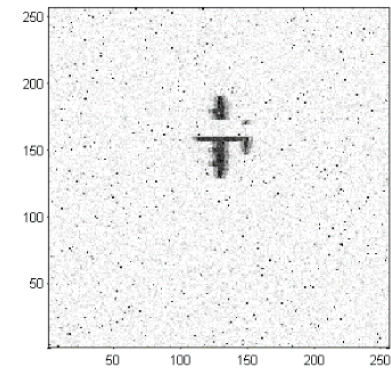

(b)
Fig. 1. Reconstructed images in presence of additive noise for the aircrafts Rafale (a) and C-130 H (b) [5].

To achieve the most accurate classification results, it is necessary for the experiments several optimization procedures to be applied to the ISAR image after its generation. It is of particular importance in this research to obtain the most detailed images ([11], [12], [13], [14], [15]), shown of figure 2 and to maintain a target-specific database with reference models [6].
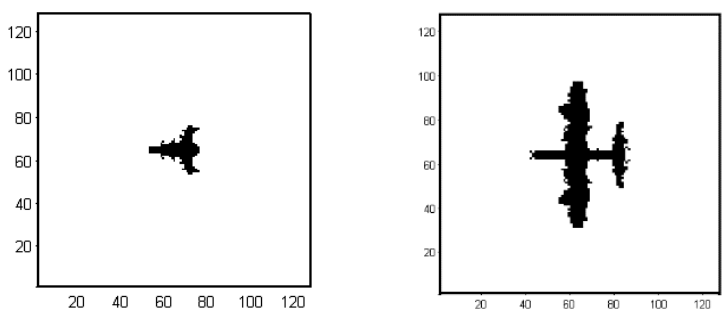

Fig. 2. Optimized ISAR images $(128 \times 128$ pixels $)$ of the aircrafts Rafale (a) and C-130 H (b).

This article proposes a method by which the observed aircraft is classified according to the location of its engines. $\mathrm{R}$ is the number of reference models to be compared. Patterns are binary matrices whose elements are a numerical representation of graphically described models of exemplary planes with different engine positions. With 
three models, the three different engine versions available, respectively, at the rear of the aircraft, on its wings and the variant where the engines are embedded in the fuselage of the airplane front or rear and do not stand out in its profile. Fig. 3 presents $9(R=9)$ exemplary graphicallydetailed solid aircraft models that form the model base in the numerical experiments. On figure 3 (a), (b), (c) are presented models with the positioning of the wing motors, in Fig. 3 (d), (e), (f) - the position of the engines at the rear of the airplane and in Fig. 1 (g), (h), (i) - engines built into the fuselage.

The object patterns are placed exactly in the middle of the frame - horizontally and vertically. Model matrices with the size $128 \times 128$ elements are formed as follows: If the pixel $(i, j)$ of the graphical solid model is part of the structure, then the corresponding element $(i, j)$ of the matrix is assigned a value of 1 , otherwise the element is 0 .

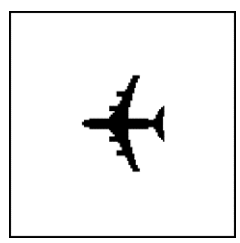

(a)

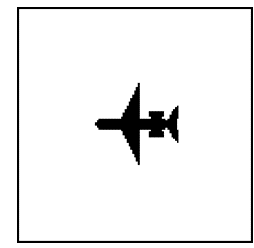

(d)

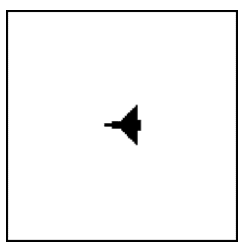

(g)

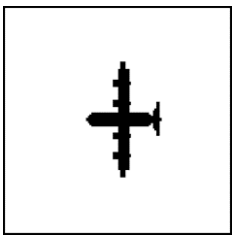

(b)

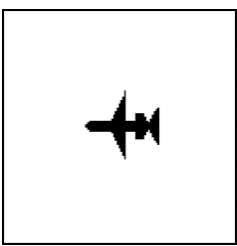

(e)

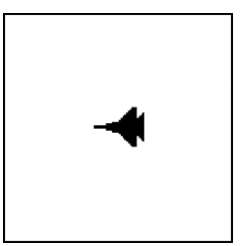

(h)

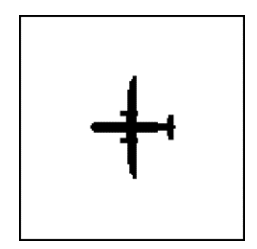

(c)

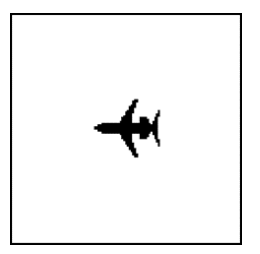

(f)

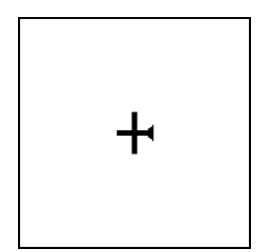

(i)
Fig.3. Graphical reference models of airplanes with engine position of types wing $(a, b, c)$, rear $(d, e, f)$ and built in fuselage $(g, h, i)$.

For each reference model, a multiplication of the binary matrix of the original image $S$ is performed with each of the $\mathrm{R}_{\mathrm{F}}(F=\overline{1,9})$ matrices.

$$
S_{m}=S R_{m}
$$

where the number of the current comparison model is $m=1, R$.

The resulting matrices $S_{m}$ will have non-zero elements only at the positions in which the $S$ and $R_{m}$ matrices have both non-zero elements. In this way, pixel address registration is performed by the matrices of a given reference model that coincide with the pixels of the image of the object being compared.The resulting $R$ counts $S_{m}$ are compared, and one of them has the maximum value. Its number corresponds to the model number (engine position) with which the object being compared has shown a maximum degree of matching between the nonzero positions of the matrices $S$ and some of the matrices $R_{m}$.

A dataset is composed of 16 aircraft models $(128 \times 128$ pixels) is used, the same like in [6] representing the planes: Eurofighter Typhoon, Pilatus 9M, Rafale, Mirage 2000, MiG-29, Gripen, Falcon 2000, F-22, F-18, F-16, C-130 H, Bombardier Q400, Boeing-747, Boeing-737, Boeing-707 and Embraer Legacy 600.

\section{Neural network design and training}

The basic idea in the proposed neural network classification approach is to compare the pixel intensity of the observed image with a set of predefined reference patterns of objects with different engine locations. In the process of realizing this approach, the task is to use the previously transformed and recognizable image in an input vector to be classified by a neural network as similar to one of the classes in the formed database.

To implement the described method, the training dataset shown on fig. 1 is used. Reference matrices containing the pixel intensity values of the reference images are converted into vectors of 16384 elements by taking the respective column elements (128x128). The resulting vectors form a common "training" matrix called Training that is designed with dimension (16384x16) - 16 columns as the number of objects in the database subject to the recognition procedure and 16384 lines as the number of pixels in 1 image.

At this stage, a matrix of the "desired result" called Target, which is needed for the neural network training process, is built too. The matrix is with dimension $3 \times 6$ - 3 rows for the three types of objects classified by their size and 6 columns, because for each type of engine position (on the wings, rear and built in the fuselage) three exemplary reference models are designed. The location of the non-zero element in the main diagonal of each column corresponds to the class number that associates the corresponding recognition result.

In the third stage of the design, in accordance with the given task, a neural architecture consisting of two layers is constructed, which is built with the tools of the programming language Matlab (fig.4) and modelled in the Simulink environment (fig.5).

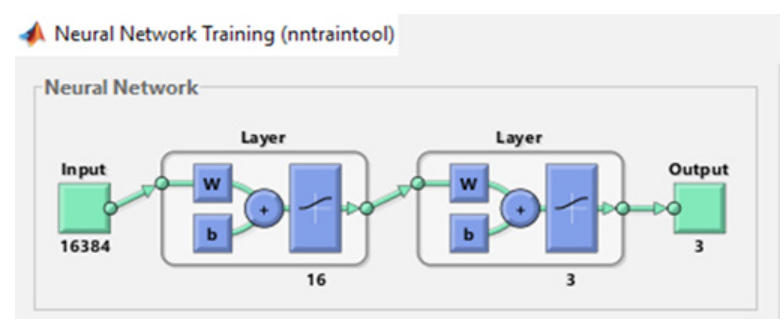

Fig.4. Neural network block diagram implemented in MATLAB. 


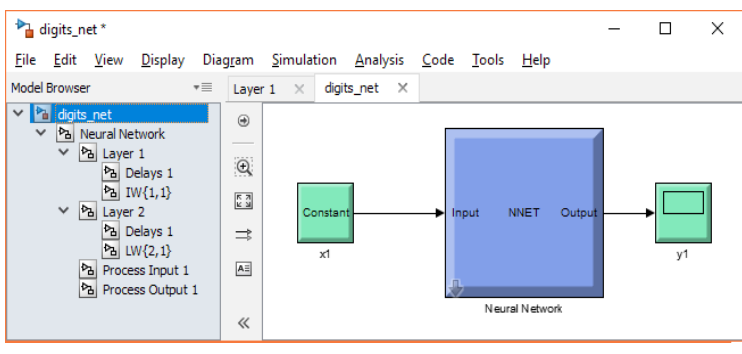

Fig.5. Schematic of Neural Network designed in Simulink.

According to the approach, the neural network is designed with backpropagation architecture of the twolayer error that performs associative memory functions in the presence of input sequence disturbances (Fig.6).

The first layer of the neural network is "hidden" and is made of 16 neurons with logarithmic-sigmoidal transmission function. These neurons form the subclasses, some of which are classified as the input vector. The inner structure of this layer is depicted in figure 7.

A delay line "Delays 1" is included in the layer structure, which converts the elements of the input sequence into an input vector.

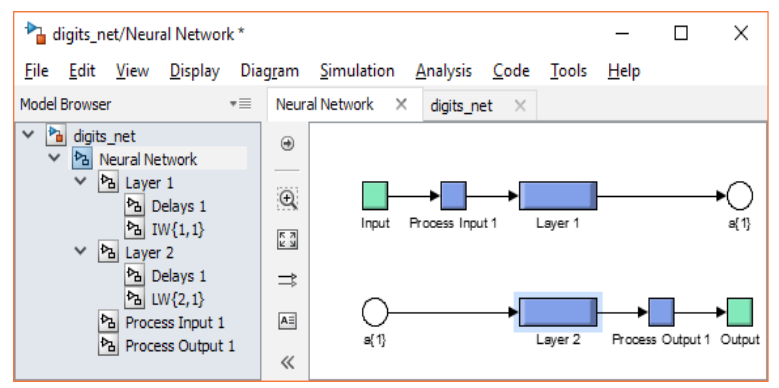

Fig. 6. Diagram of a two-layer Neural Network with right signal propagation and back propagation of the error realized in Simulink

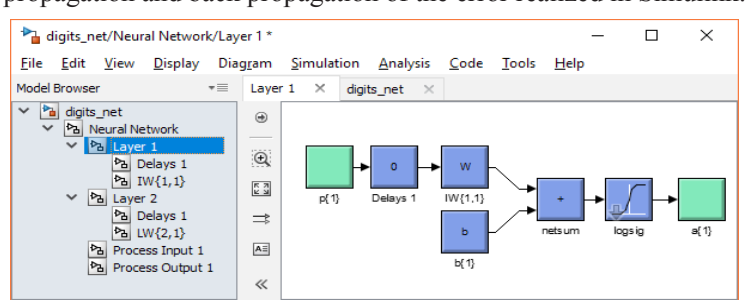

Fig.7. Structure of the first layer of Neural Network built in Simulink.

The logarithmic sigmoidal transmission function ensures high sensitivity and high resolution in the recognition process (Fig.8).

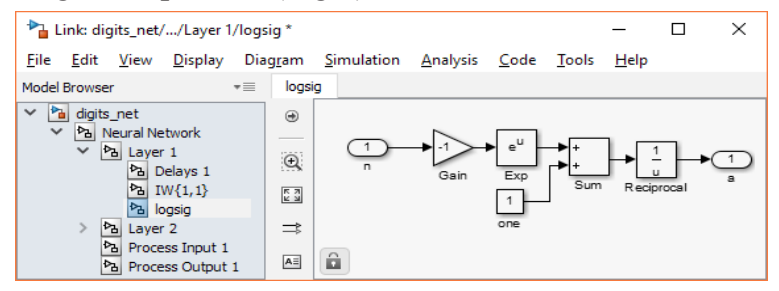

Fig.8. A structural scheme describing the mathematical model of Logarithmic-sigmoidal transfer function in Simulink.

On Figure 9 is presented the structure of the mathematical model and the physical realization of a logarithmic-sigmoidal type of function - the unfolded structure of the neuron inputs in the layer where the weight matrix IW is made by sixteen weighing vector weights whose specific values are determined at the stage of training of the neural network.

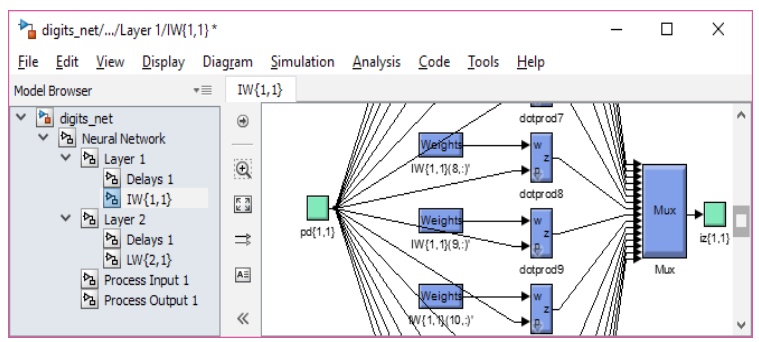

Fig.9. Structure of the input matrix of the first layer of Neural Network

The second layer contains neurons again with a logarithmic sigmoidal activation function. The number of neurons in this layer is set according to the final number of desired classes; in this case, the number is 3 . The number of the neuron "winner" will correspond to the class ("on the wings", "rear" and "built in the fuselage") to which the current input vector is determined. The role of this layer is to classify the results of the first layer and to summarize and reduce them to the user-defined number classes. Its structure is analogous to the structure of the first layer with the stated differences. On Figure 10 is presented the structure of the mathematical model and the physical realization of a logarithmic-sigmoidal type function - the unfolded structure of the neuron inputs in the layer where the weight matrix LW consists of three weight weights, the specific values of which are determined at the stage of training of the neural network.

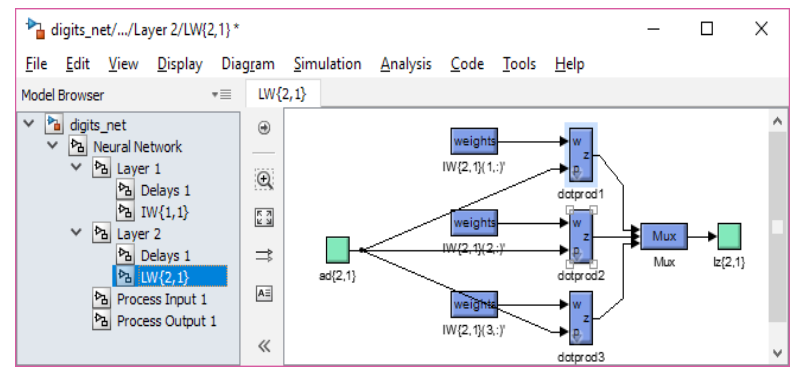
Fig.10. Structure of the input matrix of the second layer of the Neural
Network

The fourth stage of the neural architecture realization involves a neural network training process, which essentially consists of adjusting the coefficients of the weight matrices of the neurons of the two layers. Inline algorithms and procedures for automated self-learning of Matlab neural networks are used to facilitate this task. The algorithm used for learning is backpropagation of the error. The admissible error level is 0.1 , and the function of calculating this error is sse (sum squared error). The training is limited to 1000 epochs, and the results are presented in figure 11 (a). Network training is continued at higher requirements - the threshold for the permissible error level is reduced ten times to 0.01 . The learning outcomes are presented in Figure 11 (b), the training process is considered complete. 


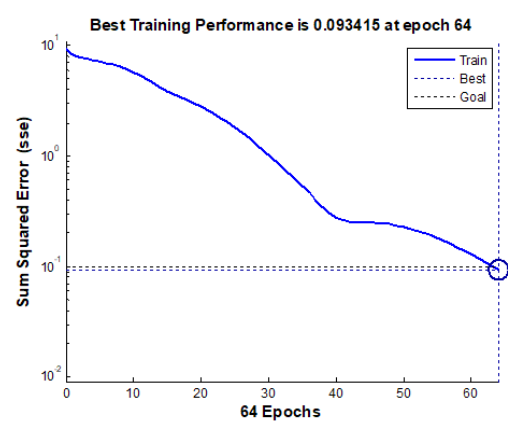

(a)

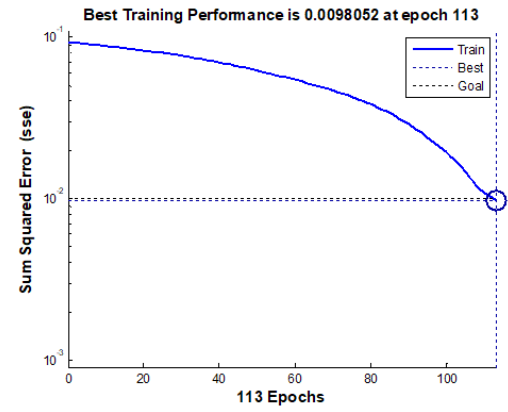

(b)

Fig.11. Graphics of learning - the desired permissible error 0.1 (a) and 0.01 (b) for noise-free learning reached for 64 and 113 epochs respectively.

\section{Results AND Discussion}

The evaluation for each object in the ISAR image is resulting in a number from 0 to 1 , expressing the classification of the airplane /by its engine location/ according the neural network design and training. The results of 3 experiments with 3 aircraft models, carried out in Matlab environment are described on table 1 . The results are improved advancing with repetitions of the experiments with the same airplane model according to the neural network's work.

Figure 12 (a) shows the graphical results of the third simulation with a $\mathrm{C} 130 \mathrm{H}$ reference model with initial parameters of the trajectory of the flying object as described in [10]. The result of the operation of the Neural Network - the position of the engines is on the wings (WingJet). The graphical results for the model Falcon 2000 (Rear Jet) and Rafale (Build In Jet) are depicted respectively on figure 12 (b) and (c).

TABLe 1 Results

\begin{tabular}{|l|c|c|c|}
\hline $\begin{array}{c}\text { Aircraft } \\
\text { model } \\
\text { used }\end{array}$ & Simul.No1 & Simul.No2 & Simul.No3 \\
\hline \multirow{3}{*}{ C-130 H } & 0.72574 & 0.793476 & 0.823917 \\
\cline { 2 - 4 } & 0.142658 & 0.06961 & 0.059254 \\
\cline { 2 - 4 } & 0.014988 & 0.019725 & 0.019126 \\
\hline \multirow{3}{*}{$\begin{array}{c}\text { Falcon } \\
\text { 2000 }\end{array}$} & 0.081169 & 0.07581 & 0.021735 \\
\cline { 2 - 4 } & 0.706703 & 0.796599 & 0.924063 \\
\hline \multirow{3}{*}{ Rafale } & 0.016546 & 0.012988 & 0.010172 \\
\cline { 2 - 4 } & 0.010236 & 0.015908 & 0.017213 \\
\cline { 2 - 4 } & 0.989968 & 0.993362 & 0.993443 \\
\hline
\end{tabular}

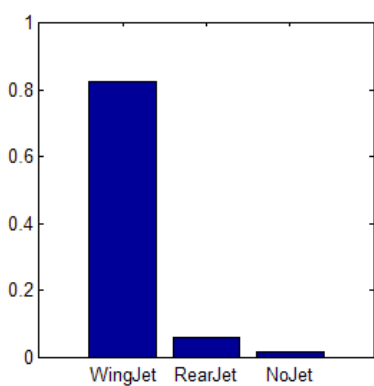

(a)

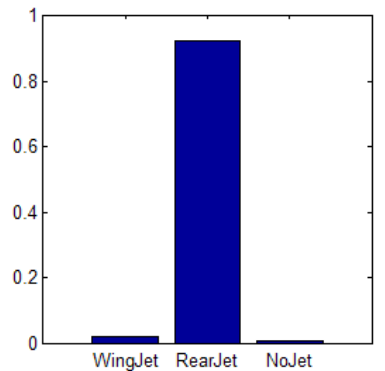

(b)

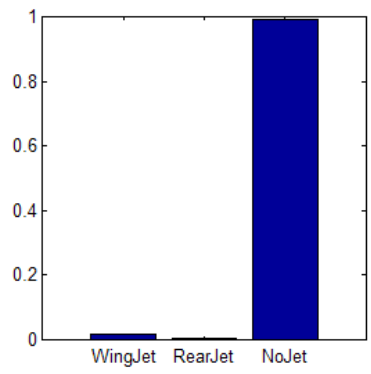

(c)

Fig.12. Neural network graphical results (for simulation № 3) for the models: C-130 H (a), Falcon 2000 (b) and the Rafale (c).

\section{CONCLUSION}

In this article, the decision-making processes concerning the type of object being identified are modelled by processing the results obtained from the proposed method. The resulting radar images can be classified into several larger groups or pluralities of planes, depending on the location of the engines, at the stage of their recognition. In this way, partial information about the observed object can be obtained even if it is not fully recognized.

The classification method can be used for any other silhouette distinguishable details in different type of aircraft structures analyzed in ISAR images. In combination with different classification approaches like speed and power detection, airflow and temperature variations etc. the problem of complete identification of an aircraft without any communication lines can achieve more grounded results.

\section{REFERENCES}

[1] Lazarov A., Minchev C., Correlation-autofocusing-spectral 2-D ISAR image reconstruction from linear frequency modulated signals, Digital Avionics Systems Conference, 2002. Proceedings, p. 27-31, Oct. 2002.

http://dx.doi.org/10.1109/DASC.2002.1052984

[2] Lazarov A. , Minchev C., ISAR Signal Modeling and Image Reconstruction with Entropy Minimization Autofocussing, 25th Digital Avionics Systems Conference, IEEE/AIAA, 2006. http:// dx.doi.org/10.1109/DASC.2006.313697

[3] Wei Q.; Hongzhong Z.; Jianxiong Z.; Qiang F., "High-Resolution Fully Polarimetric ISAR Imaging Based on Compressive Sensing", Geoscience and Remote Sensing, IEEE Transactions on (Volume:52, Issue: 10 ), 02 January 2014. http://dx.doi. org/10.1109/TGRS.2013.2295162

[4] Xiaojian Xu; Narayanan, R. M., "Three-dimensional interferometric ISAR imaging for target scattering diagnosis and modeling", Image Processing, IEEE Transactions on (Volume:10 , Issue: 7 ), Jul 2001. http://dx.doi.org/10.1109/83.931103

[5] Dimitrov D., Kirov M., Using multi-carrier probing signals for detecting non-linear objects, Scientific research and educa- 
tion in the air force -AFASES 2016, p. 247-250 http://dx.doi. org/10.19062/2247-3173.2016.18.1.33

[6] Slavyanov K., Minchev C., An algorithm for object classification procedure for ISAR images, Proceedings of 12th International Symposium on Applied Informatics and Related Areas, November 9, 2017, Székesfehérvár, Hungary, p.70-74. http://ais.amk. uni-obuda.hu/ais2017/proceedings/2017/AIS_2017_paper_17. pdf

[7] Benedetto F., Riganti Fulginei F., Laudani A., Albanese G., Automatic Aircraft Target Recognition by ISAR Image Processing based on Neural Classifier, International Journal of Advanced Computer Science and Applications, Vol. 3, No.8, pp 98-103, 2012.

http://dx.doi.org/10.14569/IJACSA.2012.030816

[8] Boulay T., Lagoutte J., Mohammad-Djafari A., Gac N., A Fuzzy-Logic Based Non Cooperative Target Recognition, Signal Image Technology and Internet Based Systems (SITIS), 2012 Eighth International Conference, Nov. 2012. http://dx.doi.org/10.1109/SITIS.2012.66

[9] Tang, N.; Gao, X.-Z. ; Li, X., "Target classification of ISAR images based on feature space optimisation of local non-negative matrix factorization", Signal Processing, IET (Volume:6, Issue: 5 ), July 2012.p. 494 - 502

http://dx.doi.org/10.1049/iet-spr.2011.0286
[10] Slavyanov K., Minchev Ch., An Algorithm of Fuzzy Inference System for ISAR Image Classification, Environment. Technology. Resources, Proceedings of the $11^{\text {th }}$ International Scientific and Practical Conference.Volume II, 154-158, http://dx.doi. org/10.17770/etr2017vol2.2599

[11] Minchev C., Slavyanov K., An opportunity for improved modeling and information analysis in ISAR systems, Machines. Technologies. Materials., Vol. 9 (2015), Issue 6, p. 24-27 https:// stumejournals.com/journals/mtm/2015/6/24

[12] Parmar J.; Patil S."Performance evaluation and comparison of modified denoising method and the local adaptive wavelet image denoising method", Intelligent Systems and Signal Processing (ISSP), 2013 IEEE http://dx.doi.org/10.1109/ISSP.2013.6526883

[13] Russo F., "A fuzzy approach to performance measurement of grayscale image denoising algorithms", Intelligent Signal Processing (WISP), 2015 IEEE http://dx.doi.org/10.1109/ WISP.2015.7139159

[14] Otsu N., A Threshold Selection Method from Gray-Level Histograms, IEEE Trans., Systems, Man, and Cybernetics, vol.9, no.1, p.62-66, 1979. http://dx.doi.org/10.1109/TSMC.1979.4310076

[15] Minchev C., Slavyanov K., An algorithm for ISAR image optimization procedure, Bulgaria, National Military University, Proceedings of Internatinal scientific conference, 2015, ISSN 2367-7902, p.177-184. http://www.aadcf.nvu.bg/scientific events/papers/NS_2015.pdf 